\title{
A Hollow-Core Resonator Fiber Optic Gyroscope Using Nodeless Anti-Resonant Fiber
}

\author{
Glen A. Sanders ${ }^{1,4}$, Austin A. Taranta ${ }^{2,5}$, Chellappan Narayanan ${ }^{1}$, Eric \\ Numkam Fokoua ${ }^{2}$, Seyedmohammad Abokhamis Mousavi ${ }^{2}$, Lee K. \\ StRANDJORD ${ }^{3}$, MARC SMICIKLAS ${ }^{1}$, ThOMAS D. BRADLEY ${ }^{2}$, JOHN HAYES ${ }^{2}$, GREGORY \\ T. Jasion ${ }^{2}$, Tiequn QIU ${ }^{1}$, Wes Williams ${ }^{1}$, Francesco Poletti ${ }^{2}$, David $\mathbf{N}$. \\ PAYNE $^{2}$ \\ ${ }^{1}$ Honeywell International, 21111 N. 19th Ave, Phoenix, AZ USA 85027 \\ ${ }^{2}$ Optoelectronics Research Centre, University of Southampton, SO171BJ, UK3 \\ ${ }^{3}$ Honeywell International, 12001 State Highway 55, Plymouth, MN USA 55441 \\ 4Glen.A.Sanders@honeywell.com \\ ${ }^{5}$ A.A.Taranta@soton.ac.uk
}

Resonator Fiber-Optic Gyroscope (RFOG) performance has hitherto been limited by non-linearity, modal impurity, and backscattering in the sensing fibers. The use of hollow-core fiber (HCF) effectively reduces non-linearity, but the complex interplay among glass and air-guided modes in conventional HCF technologies can severely exacerbate RFOG instability. By employing high-performance nested anti-resonant nodeless fiber, we demonstrate long-term stability in a hollow-fiber RFOG of $0.05 \mathrm{deg} / \mathrm{hr}$, nearing the levels required for civil aircraft navigation. This represents a 3X improvement over any prior hollow-core RFOG and a factor of 500X over any prior result at integration times longer than 1 hour. (C) 2020 Optical Society of America

\section{Introduction}

The Resonator Fiber Optic Gyroscope (RFOG) has great potential as a next-generation guidance and navigation technology. This is because it combines the sensitivity advantages of multi-turn coils used in interferometric fiber optic gyros with the enhancements afforded by the recirculation of light in a resonant cavity. Over a decade ago, the use of hollow-core fiber $(\mathrm{HCF})$ in the resonator sensing path was proposed to overcome many of the historical drift-mechanisms and barriers attributed to the employment of monochromatic light in conventional glass-core fibers [1]. Despite the appeal of this concept, practical problems - mostly linked to insufficient modal purity in the fiber - have until recently prevented demonstrations of an HCF-based RFOG with good performance. In this paper, we present, for the first time, the use of single-mode, anti-resonant nodeless HCF in an RFOG and show that the excellent properties of the fiber enable us to measure long-term bias stability down to $0.05 \mathrm{deg} / \mathrm{hr}$ over 1-10 hours. This represents a factor of 3x improvement over the best previously reported result [2], and is achieved over the multi-hour integration times required for inertial navigation. For integration times longer than 1 hour, this represents an improvement of more than 500X This stability highlights the attractive properties of nodeless antiresonant fibres in resonator applications and brings the performance of HCF-based RFOGs to within an order of magnitude of that required for civil aircraft navigation.

In the RFOG, rotation rate is measured by determining resonant frequency shifts between the clockwise (CW) and counterclockwise (CCW) light propagation in a multi-turn passive fiber ring resonator [3,4], as shown in Fig 1. In this arrangement, CCW and CW laser waves at fo and fo $+\Delta \mathrm{f}$ are precisely tuned to the centers of CCW and CW resonances of a fiber ring resonator to determine their resonance frequencies. In the presence of rotation, the resonances are shifted, and the laser input frequency difference, $\Delta \mathrm{f}$, is proportional to rotation rate $\Omega$, via $\Delta \mathrm{f}=(\mathrm{D} / \mathrm{n} \lambda)^{*} \Omega$, where $\mathrm{D}$ is the coil diameter, $\lambda$ the wavelength, and $n$ is the refractive index of the fiber core.

The use of a high power, narrow linewidth laser to interrogate the high-finesse resonator can present significant challenges for conventional solid-core fibers, as both linear and non-linear interactio ns between the optical field and the glass produce a multitude of spurious 


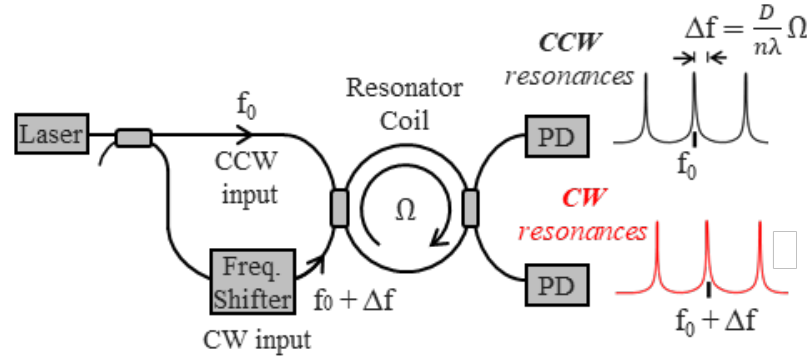

Fig 1. Basic RFOG principle of operation. Laser inputs for probing resonance frequency shifts due to rotation can by realized by a single laser, plus a frequency-shifter, or two separate phase-locked lasers.

signals, such as stimulated Brillouin scattering Kerr nonlinearity [5], coherent backscatter [6], and polarization effects [7,8]. These give rise to an erroneous indication of rotation rate, or bias instability. The nonlinear effects impose optical power limits (lowering SNR), and tight power control requirements, while in the latter two cases, use of a narrow linewidth laser allows light in potentially a multitude of spurious paths to be fully coherent with signal light traversing the resonator. Thus, polarization effects and backscatter impose tight constraints on the resonator fiber, since distributed backscattering or modal couplings at disparate and distant sites along the fiber can interfere coherently. Because these spurious signals are dependent on minute environmental perturbations, they can severely compromise RFOG bias stability.

The ideal waveguide for an RFOG is thus one in which the optical field is confined in a low nonlinearity material such as air or vacuum (i.e. an $\mathrm{HCF}$ ), and in which only a single spatial mode is guided with low loss, low polarization crosstalk, and low backscatter [1]. The first demonstration of HCF in aresonator for rotation sensing used a HollowCore Photonic Bandgap Fiber (HC-PBGF) with free-space beamsplitters for the input/output optics and achieved a finesse of 42 [1]. Digonnet and colleagues demonstrated that HC-PBGFs could be used to reduce nonlinearity and environmental sensitivity in an interferometric fiber gyro (IFOG) [9], and reported the first measured performance of an RFOG based on HC-PBGF, with a long-term stability of $1800 \mathrm{deg} / \mathrm{hr}$ at an integration time, $\tau$, of 1 hour [10]. By using apolarization maintaining HC-PBGF and a hybrid solid/hollow resonator, Yan et al. improved this to $25 \mathrm{deg} / \mathrm{hr}$ stability [2] at $\tau \approx 0.1 \mathrm{hr}$ [11]. More recently, Suo et al. [2] demonstrated the beneficial aspects of anti-resonant guiding HCF, showing $0.15 \mathrm{deg} / \mathrm{hr}$ short-term $(\tau \approx 200 \mathrm{sec})$ bias instability in a transmission-mode RFOG using Kagome HCF. Our own observations of modal-purity problems in HC-PBGFs having nodal points, reports of higher backscatter (vs. solid single-mode fiber), and spurious glasspropagating modes in these fibers $[12,13]$ led us to investigate an alternative $\mathrm{HCF}$ type

Here we focus on Nested Anti-resonant Nodeless Fiber (NANF) [14], the structure of which is compared in Fig 2 to that of a more tradition al HC-PBGF. In HC-PBGFs a lattice of glass nodes (the vertices of the hexagonal cells shown in Fig 2a) establishes an air-guiding bandgap in the core. However, the glass struts which are required to position these nodes at the correct locations in space increase the loss, reduce the bandwidth, and affect the modal and polarization properties of the fiber [15], as hinted by the irregular spatial arrangement of field contours near the core boundary in Fig 2a Moreover, they support glass-propagating surface modes [15], which can contribute to RFOG drift. In contrast, for NANFs it is the struts themselves - in the form of the non-contacting, nested tubes (Fig 2b) - which confine light by coherent reflection. Only wavelengths which are resonant with the membrane thickness can couple out of the central void. States elsewhere in the spectrum are said to be in anti-resonance and are prevented from traversing the glass membranes in a manner akin to a Fabry-Pérot etalon.

(a)

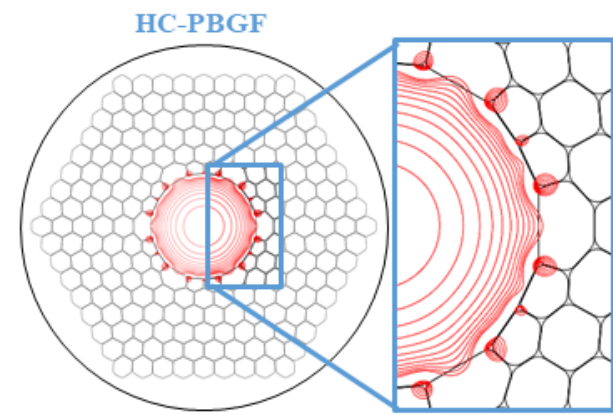

NANF

(b)

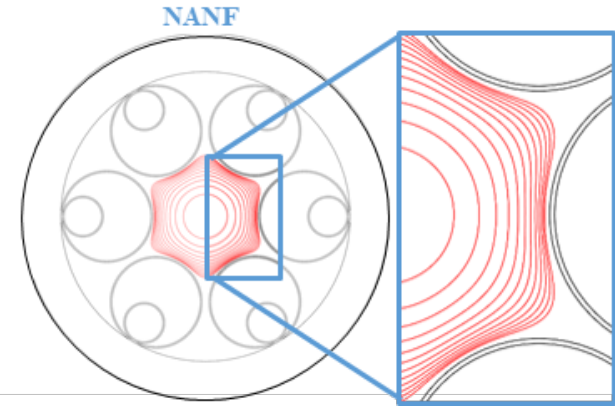

Fig 2. Comparison of HC-PBGF (a) and NANF (b) structures. Black lines show air/glass boundaries. Red lines are contours of equal optical power density from our model. Power localized at core-boundary nodes of the HC-PBGF (shown as red dots) are surface modes.

Along with confinement, this mechanism produces an exceptionally low field overlap with the glass. For suitably designed NANFs, the total power propagating in the glass at anti-resonance varies from 1060ppm, a factor of $>10 \mathrm{x}$ less than in HC-PBGFs [15], thus reducing the fiber nonlinearity proportionally. Moreover, the field at the core boundary has excellent spatial regularity, with no surface modes and thus less polarization and spatial mode coupling [16]. Additionally, NANF cladding tubes can be designed to strongly attenuate all the airguided higher-order modes (HOMs) of the core [17], rendering NANFs effectively single-mode after sufficient propagation length. Finally, NANFs have recently achieved the lowest loss of all HCF technolo gi es (028 dB/km, [18]), and can be tailored for guidance in multiple spectral regions. This combination of ultra-low non-linearity, modal purity, and low loss promises to make NANFs an attractive enabler for RFOG.

\section{Fiber Design \& Performance}

The two fibers tested in this work are depicted in Fig. 3. Both are 6 nested tube designs with $33 \mu \mathrm{m}$ core diameters. The slightly nonuniform micro-structure of NANF 1 (Fig 3a) gives rise to a higher loss at $1550 \mathrm{~nm}$ of $20 \mathrm{~dB} / \mathrm{km}$, while the more recently produced NANF 2 (Fig. 3b) shows an improved symmetry and $2.8 \mathrm{~dB} / \mathrm{km}$ loss. Both fibers were fabricated via a standard stack and draw process and they share similar design parameters with the low-loss $(13 \mathrm{~dB} / \mathrm{km})$ NANF reported in [19]. The membrane thicknesses of NANF 1 and NANF 2 are $1.068 \pm 0.061 \mu \mathrm{m}$ (mean $\pm 1 \sigma$ ) and $1.22 \pm 0.037 \mu \mathrm{m}$ respectively.

One of the key challenges to HCF deployment in an RFOG lies in coherent backscattering. Judicious signal processing techniques can alleviate the primary error mechanisms that arise, but are unable to suppress secondary backscatter errors, including those mediated by surface modes in HC-PBGFs. Terrel et al. [10], showed that HC-PBGF backscatter severely limits the bias instability of an HCF RFOG. There, the HC-PBGF coil showed a recaptured backscatter factor on the order 
of $10^{-6} \mathrm{~m}^{-1}$ - at least 25 times greater than that of solid-core SMF. Reflectometry measurements show that backscattering is dramatic ally reduced in NANFs, with the two fibers tested showing backscatter at least an order of magnitude lower than that of solid-core fiber. As shown in the high-resolution optical time domain reflectometry trace of Fig. 3c, NANF 2 has a backscattered power at or below the noise floor of the instrument, which is $12 \mathrm{~dB}$ below the Rayleigh scattering floor of SMF28. Only a few discrete peaks are visible in the trace, and even the worst of these is still $\sim 5 \mathrm{~dB}$ lower than this solid fiber limit.
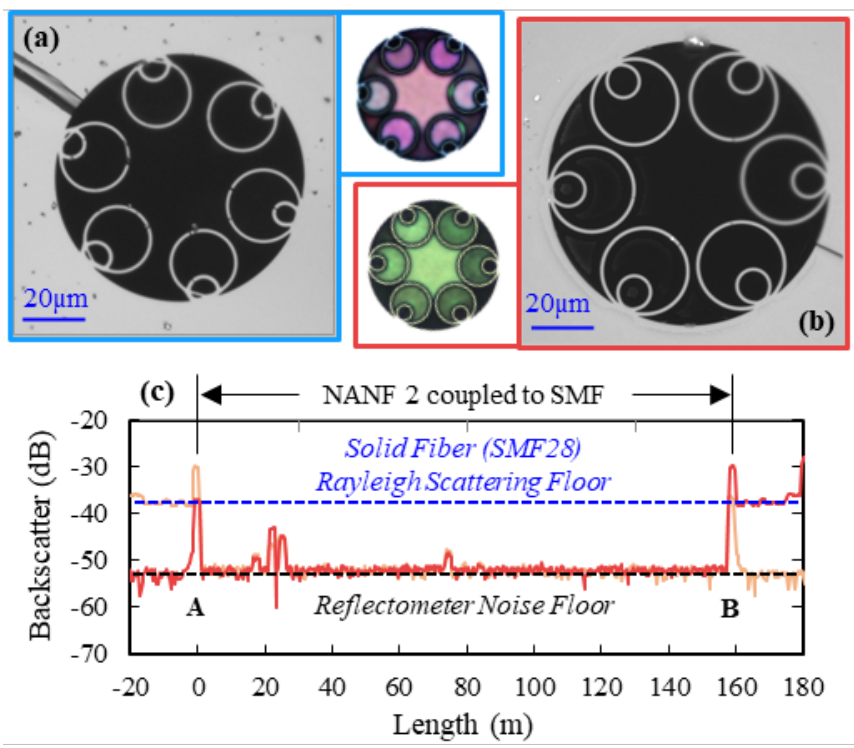

Fig 3. Microstructure geometry and fiber-level performance: Optical microscope images of (a) NANF 1 and (b) NANF 2 (true-color images show illumination of the core region with white light), (c) Bi-direction nal reflectometry trace (OTDR) on NANF 2 at $1550 \mathrm{~nm}$.

In NANF 1, cutback measurements using spatial and spectral imaging ("S2 imaging" [20]) indicate the least attenuated HOM, LP11, shows $>1280 \mathrm{~dB} / \mathrm{km}$ loss, and thus the highest HOM power after traversing the full coil is $160 \mathrm{~dB}$ below that of the fundamental. Bi-directional $\mathrm{S}^{2}$ imaging on the full length of NANF 2, shows the presence of a modal coupling point $\sim 25 \mathrm{~m}$ from the " $\mathrm{A}$ " side of the fiber, which we attribute to a localized scattering defect as this matches the reflective defect in the OTDR trace (Fig 3c). Excluding this defect, for an optimized launch, all modal interferences are attenuated to below $-85 \mathrm{~dB}$, the noise floor of the measurement. This represents a remarkable improvement over HC-PBGFs, where discrete mode couplings are orders of magnitude larger, and in which surface mode interactions produce a persistent, distributed modal interference background [13].

\section{RFOG Configuration \& Performance}

Both NANFs 1 and 2 were deployed in the experimental RFOG shown in Fig 4. In the absence of suitable fiber couplers using HCF, free space optical lenses [1] were used to couple each end of the $136 \mathrm{~m}$ NANF coil to a first and second PM solid-core fiber coupler. The two PM couplers were then spliced together to close the resonator loop, and to provide means for coupling light into, and out of, the resonator. Inside each freespace portion of the resonator a half-wave plate was used to align the optical polarization state to the principal axis of the fiber coil, and a polarizer was used to reduce polarization errors in the gyro [7]. The coil was wound around a 4.5-inch diameter cylinder. The coupling loss of each free-space to fibre coupling was approximately $0.65 \mathrm{~dB}$, and for each leg the 90/10 coupler split ratios resulted in a total resonator loss of $2.18 \mathrm{~dB}$ and a finesse of approximately 14.5. The CW and CCW resonances of the resonator were probed by lasers from a MultiFrequency Laser Source (MFLS), having very low relative phase noise between CW and CCW input light. This technique [21], uses a master laser diode that is tightly locked to the $\mathrm{CW}$ resonance at the $\mathrm{CW}$ reflection port using a Pound Drever Hall (PDH) loop. Slave lasers 1 and 2 are phase-locked to the master laser, each with a tunable frequency offset that allows resonance tracking loops to adjust their optical carrier frequencies, at fcw and $\mathrm{fccw}_{\mathrm{w}}$, respectively, to the $\mathrm{CW}$ and CCW resonances. Light from slave lasers 1 and 2 is detected at CW and CCW transmission detectors after circulating within the resonator and being transmitted out of the resonator by an input/output coupler. Light emanating from each transmission port is directed to a transmission port detector via a circulator.
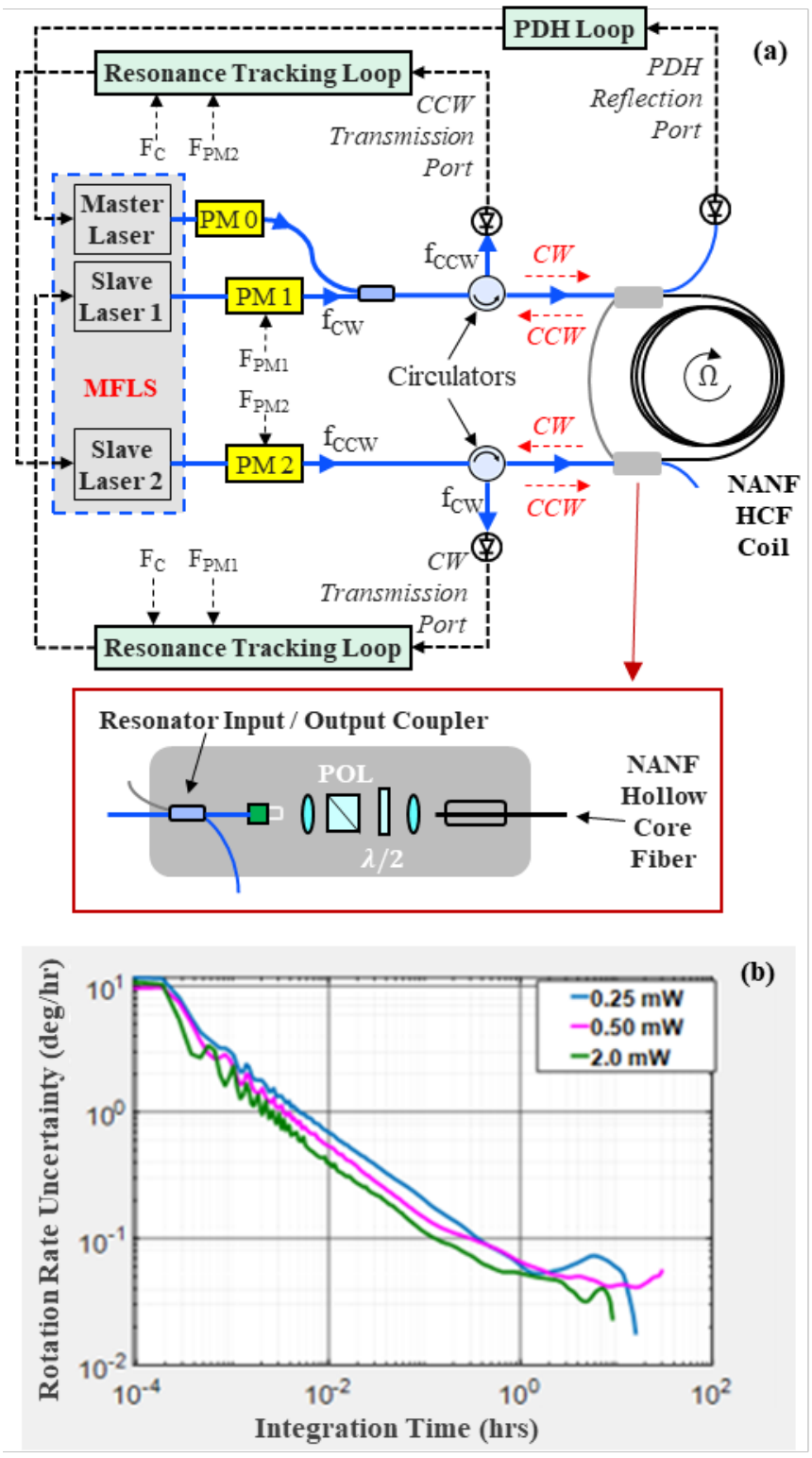

Fig 4. (a) RFOG arrangement using NANF fiber for the resonator coil (b) Drift-rate Allan Deviation of the RFOG plotted for 3 different resonator input optical power levels. The long-term bias instability for integration times of $1-10 \mathrm{hrs}$ is roughly $0.05 \mathrm{deg} / \mathrm{hr}$. 
The resonance tracking loops are based on detection of the resonance using a modulation and demodulation approach described in [22]. Slave lasers 1 and 2 are modulated at different frequencies, FPM1 in PM1 and FPM2 in PM2, and subsequently demodulated in the resonance tracking loops as a means of separating signal light from backscattered light [6]. A second, common modulation, Fc (not shown) is applied to both lasers, and after the detector signals are demodul at ed at FPM1 and FpM2, each of them is demodulated at Fc. This double demodulation scheme precisely determines the cavity resonance frequencies, while suppressing backscatter errors.

To measure the performance of the RFOG of Fig 4(a), the setup was mounted on a stable pier at room temperature $\left(\sim 22^{\circ} \mathrm{C}\right)$ with its input rotation rate being only a fraction of Earth's rate, or about $8.2 \mathrm{deg} / \mathrm{hr}$. At such low and stable input rate, any drift or variation can be attributed to random noise at short integration time, or bias instability at longer integration time. The rate output variation of the gyro (rate uncertain ty) as a function of integration time (Allan Deviation) is plotted in Fig. 4(b), for the case of the NANF 2 coil. Data were taken with three different power levels at the entrance to the resonator over runs of 20 to 60-hour duration. For integration times, $\tau$, less than $10^{-1} \mathrm{hrs}$, the rate uncertainty is limited by random noise, as evidenced by the $\tau^{1 / 2}$ dependence. The rate uncertainty no longer improves as $\tau^{-1 / 2}$ in the 1-10 hours region, i.e. showing bias instability of the gyro. For all three curves the rate uncertainty reaches a floor at $\sim 0.05 \mathrm{deg} / \mathrm{hr}$. Also, within the (inherently growing) error bars for $\tau>1 \mathrm{hr}$, the bias stability is relatively constant across a wide range (8x) of optical power from $025 \mathrm{~mW}$ to $2 \mathrm{~mW}$. This observation is consistent with the low non-linearity expected for NANF.

\section{Conclusions}

We have demonstrated the first use of a NANF HCF in an RFOG. The considerably improved spatial-mode purity and backscattering of the fiber over photonic bandgap HCFs are crucial to our observation of record low bias drift performance, which is perhaps the keystone metric of technological advancement in RFOGs. Long-term bias stability was roughly $0.05 \mathrm{deg} / \mathrm{hr}$ for observation times of $1-10 \mathrm{hrs}$, more than $100 \mathrm{X}$ longer integration time than prior bias-stability demonstrations on HCF-based RFOGs. Stability was largely independent of optical power, consistent with low non-linearity in NANF HCF. These encouraging results show an improvement of 3x over any previously reported HCFbased RFOGs and they represent a 500X improvement over prior longterm measurements. As this is a free-space implementation, refinements to the gyro packaging are expected to further improve performance for subsequent embodiments. Nonetheless, this work demonstrates performance which is within about $10 \mathrm{x}$ the requirem ent for civil aircraft navigation usage, which we believe is realistically achievable through further optimization of the RFOG and this promising relatively young fiber technology.

Funding. This project has received funding from the European Research Council (ERC) (grant agreement $n^{\circ}$ 682724).

Acknowledgements. ENF gratefully acknowledges support from a Royal Academy of Engineering Research Fellowship.

Disclosures. The authors declare no conflicts of interest.

\section{References}

1. G. A. Sanders, L. K. Strandjord, and T. Qiu, "Hollow Core Fiber Optic Ring Resonator for Rotation Sensing," in Optical Fiber Sensors (OSA, 2006), p. ME6.

2. X. Suo, H. Yu, J. Li, and X. Wu, "Transmissive resonant fiber-optic gyroscope employing Kagome hollow-core photonic crystal fiber resonator," Opt. Lett. 45, 2227 (2020).
3. S. Ezekiel and S. R. Balsamo, "Passive ring resonator laser gyroscope," Appl. Phys. Lett. 30, 478-480 (1977).

4. D. M. Shupe, "Fiber resonator gyroscope: sensitivity and thermal nonreciprocity.," Appl. Opt. 20, 286-289 (1981).

5. K. Iwatsuki, K. Hotate, and M. Higashiguchi, "Kerr effect in optical passive ring-resonator gyros," in Optical Fiber Sensors (OSA, 1985), Vol. L, p. ThGG13.

6. K. Iwatsuki, K. Hotate, and M. Higashiguchi, "Effect of Rayleigh backscattering in an optical passive ring-resonator gyro," Appl. Opt. 23, 3916 (1984)

7. K. Iwatsuki, K. Hotate, and M. Higashiguchi, "Eigenstate of polarization in a fiber ring resonator and its effect in an optical passive ring-resonator gyro," Appl. Opt. 25, 2606 (1986)

8. L. K. Strandjord and G. A. Sanders, "Resonator fiber optic gyro employing a polarization-rotating resonator," in Fiber Optic Gyros: 15th Anniversary Conf, S. Ezekiel and E. Udd, eds. (1992), Vol. 1585, pp. 163-172.

9. M. Digonnet, S. Blin, H. K. Kim, V. Dangui, and G. Kino, "Sensitivity and stability of an air-core fibre-optic gyroscope," Meas. Sci. Technol. 18, 3089-3097 (2007).

10. M. A. Terrel, M. J. F. Digonnet, and S. Fan, "Resonant Fiber Optic Gyroscope Using an Air-Core Fiber," J. Light. Technol. 30, 931-937 (2012).

11. Y. Yan, H. Ma, L. Wang, H. Li, and Z. Jin, "Effect of Fresnel reflections in a hybrid air-core photonic-bandgap fiber ring-resonator gyro," Opt. Express 23, 31384 (2015).

12. M. J. F. Digonnet and J. N. Chamoun, "Recent developments in laserdriven and hollow-core fiber optic gyroscopes," Fiber Opt. Sensors Appl. XIII 9852, 985204 (2016).

13. G. A. Cranch and G. A. Miller, "Coherent light transmission properties of commercial photonic crystal hollow core optical fiber," Appl. Opt. 54, F8 (2015).

14. F. Poletti, "Nested antiresonant nodeless hollow core fiber," Opt. Express 22, 23807 (2014)

15. F. Poletti, M. N. Petrovich, D. J. Richardson, and M. J. Li, "Hollowcore photonic bandgap fibers: Technology and Applications," Nanophotonics 2, 315-340 (2013).

16. A. Taranta, E. Numkam Fokoua, S. Abokhamis Mousavi, J. R. Hayes, T. D. Bradley, G. T. Jasion, and F. Poletti, "Exceptional polarization purity in antiresonant hollow-core optical fibres," Nat. Photonics (2020).

17. P. Uebel, M. C. Günendi, M. H. Frosz, G. Ahmed, N. N. Edavalath, J.M. Ménard, and P. S. J. Russell, "Broadband robustly single-mode hollow-core PCF by resonant filtering of higher-order modes," Opt. Lett. 41, 1961 (2016).

18. G. T. Jasion, T. D. Bradley, K. Harrington, H. Sakr, Y. Chen, E. N. Fokoua, I. A. Davidson, A. Taranta, J. R. Hayes, D. J. Richardson, and F. Poletti, "Hollow Core NANF with $0.28 \mathrm{~dB} / \mathrm{km}$ Attenuation in the C and L Bands," in Optical Fiber Communication Conference Postdeadline Papers 2020 (OSA, 2020), p. Th4B.4.

19. T. D. Bradley, J. R. Hayes, Y. Chen, G. T. Jasion, S. R. Sandoghchi, R. Slavik, E. N. Fokoua, S. Bawn, H. Sakr, I. A. Davidson, A. Taranta, J. P. Thomas, M. N. Petrovich, D. J. Richardson, and F. Poletti, "Record Low-Loss 1.3dB/km Data Transmitting Antiresonant Hollow Core Fibre," in 2018 European Conference on Optical Communication (ECOC) (IEEE, 2018), Vol. 2018-Septe, pp. 1-3.

20. J. W. Nicholson, L. Meng, J. M. Fini, R. S. Windeler, A. DeSantolo, E. Monberg, F. DiMarcello, Y. Dulashko, M. Hassan, and R. Ortiz, "Measuring higher-order modes in a low-loss, hollow-core, photonicbandgap fiber," Opt. Express 20, 20494 (2012).

21. J. Wu, M. Smiciklas, L. K. Strandjord, T. Qiu, W. Ho, and G. A. Sanders, "Resonator fiber optic gyro with high backscatter-error suppression using two independent phase-locked lasers," in 24th International Conference on Optical Fibre Sensors, H. J. Kalinowski, J. L. Fabris, and W. J. Bock, eds. (2015), p. 963410.

22. L. K. Strandjord, T. Qiu, M. Salit, C. Narayanan, M. Smiciklas, J. Wu, and G. A. Sanders, "Improved Bias Performance in Resonator Fiber Optic Gyros using a Novel Modulation Method for Error Suppression," in 26th International Conference on Optical Fiber Sensors (OSA, 2018), p. ThD3. 